\title{
Molecularly Engineered PEG-TRAP Conjugates for Platelet Activation and Accelerated Hemostasis
}

\author{
Xiao-Hua Qin ${ }^{\dagger * *}$, Jie Chen ${ }^{\dagger}$, Krystyna Labuda ${ }^{\dagger}$, Veronika Hruschka ${ }^{\dagger}$, Robert Liska ${ }^{\ddagger}$, Heinz Redl ${ }^{\dagger}$, Paul Slezak ${ }^{\dagger *}$ \\ †Ludwig Boltzmann Institute for Traumatology in coop. AUVA, Donaueschingenstrasse 13, 1200 Vienna, Austria \\ $\ddagger$ Vienna University of Technology, Institute of Applied Synthetic Chemistry, Getreidemarkt 9, 106o Vienna, Austria \\ \#ETH Zurich, Institute for Biomechanics, Leopold-Ruzicka-Weg 4, 8093 Zurich, Switzerland \\ KEYWORDS. Polymer-peptide conjugates, platelet activation, thrombin receptor-activating peptide (TRAP), hemostasis, blood \\ coagulation
}

\begin{abstract}
Synthetic peptide conjugates that can molecularly target and activate blood platelets are designed for hemostasis applications. An engineered thrombin receptor-activating peptide (TRAP-Cys, SFLLRNPNC) is covalently attached to cell-compatible 8-arm poly (ethylene glycol) maleimides via Michael addition. It is proved that the ability of TRAP to activate platelets is retained in the conjugated form by means of thromboelastometry, platelet aggregation assay and flow cytometry. A $0.5 \%$ PEG-TRAP solution can efficiently activate platelets and shorten the clotting time to $\sim 45 \%$.
\end{abstract}

The ability to rapidly stop bleeding is crucial for life-saving in surgical and traumatic injuries. ${ }^{1}$ However, existing hemostatic strategies ${ }^{2}$ are often based on blood components such as fibrinogen and thrombin, which are expensive and unstable in liquid form. ${ }^{3}$ Designing alternative hemostatic materials ${ }^{4-6}$ with improved properties is therefore of paramount significance for modern medical applications.

Platelets are key players in the process of blood coagulation. ${ }^{7}$ In the primary stage of hemostasis, platelet activation is a crucial event that contributes to platelet aggregation, fibrin polymerization and formation of clots at the injury site. Notably, the serine protease thrombin is one of the most potent agonist for platelets. ${ }^{8}$ The cellular effects of thrombin are mediated by protease-activated receptors (PARs) .9 For instance, PAR-1 is highly expressed in human platelets and the principle of thrombin-mediated PAR-1 signaling is depicted in Scheme 1. After proteolytic cleavage by thrombin, a new $\mathrm{N}$-terminus is exposed, which acts as a tethered ligand to trigger platelet activation. In 1992, Scarborough et al. ${ }^{10}$ reported that a key functional unit in the ligand is a SFLLRN- amino acid sequence, also termed as the thrombin receptor-activating peptide (TRAP). TRAP has been used as soluble agonist in clinical-relevant assays to analyze platelet functions.


Scheme 1. Design of TRAP-polymer conjugates: A, principle of thrombin-triggered platelet activation: after proteolysis by thrombin (1), the new N-terminus binds to the second loop of the PAR-1 receptor (2), which triggers platelet activation (3). After activation, growth factors and cytokines are released from platelets to promote wound repair (4). B-C, Schematics of the preparation of PEG-8-TRAP conjugates via thiol-maleimide conjugation.

In a previous study, we reported the design and synthesis of synthetic hemostats based on polyvinyl alcohol (PVA)TRAP conjugates via radical-mediated thiol-norbornene photo-conjugation. " Since UV-irradiation might induce undesirable effects on peptides, here we describe an alternative approach to prepare polymer-TRAP conjugates via thiol-maleimide conjugation. This reaction has been exploited to rapidly form synthetic hydrogels and even for in situ $3 \mathrm{D}$ cell encapsulation due to its high efficiency ${ }^{12}$ and good cell-compatibility. ${ }^{13}$

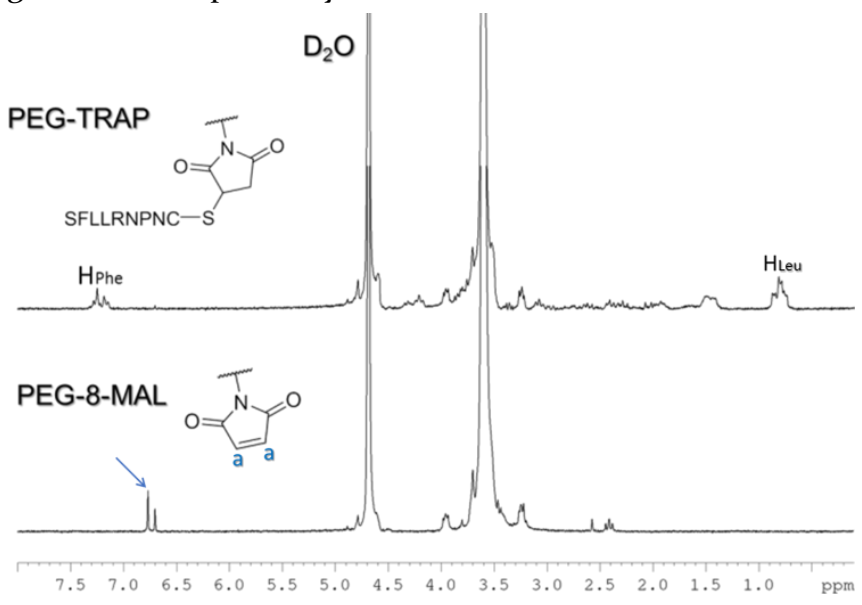

Figure 1. ${ }^{1} \mathrm{H}-\mathrm{NMR}\left(\mathrm{D}_{2} \mathrm{O}\right)$ spectra of PEG-TRAP conjugates and PEG-8-MAL precursors. 
To prepare the conjugates, a Cysteine-containing TRAP derivative (SFLLRNPNC, TRAP-Cys) was prepared through custom solid-phase peptide synthesis. TRAP-Cys was reacted with eight-arm poly(ethylene glycol) maleimides (PEG-8-MAL) in an off-stoichiometry (thiol : MAL = 1.2:1) under the catalysis of triethanolamine in PBS buffer for $1 \mathrm{~h}$. The conjugates were subsequently purified via dialysis in deionized water to remove unreacted peptides. ${ }^{1} \mathrm{H}-\mathrm{NMR}$ spectra (Figure 1) show that the unsaturated proton signals in PEG-8-MAL at $\sim 6.7$ Ppm disappeared after the conjugation with TRAP-Cys. Furthermore, proton signals corresponding to the phenylalanine $(\mathrm{F})$ and leucine $(\mathrm{L})$ residues in TRAP-Cys were observed in the PEG-TRAP. HPLC analysis showed that $>90 \%$ peptides were removed after the purification. These data suggest that maleimide groups in PEG-8-MAL reacted with TRAP-Cys peptides at high efficiency via off-stoichiometric Michael-addition reaction.

We further tested the cytotoxicity of the PEG-8-MAL precursor solutions against $\mathrm{C}_{2} \mathrm{C}_{12}$ myoblasts using a lactate dehydrogenase (LDH) assay. Unexpectedly, the solutions of as-received PEG-8-MAL induced remarkable decrease of cell activity after $24 \mathrm{~h}$ cultivation (not shown). The observed toxic effects are likely due to the maleic anhydride residues in the preparative procedure of PEG-8-MAL. Therefore, all the PEG-8-MAL precursors were purified via dialysis for further biological tests. The dialyzed PEG-8MAL (Figure Si) exhibited negligible cytotoxic effects at varying weight concentrations after cultivation for $48 \mathrm{~h}$, indicating their excellent cytocompatibility. Further toxicity studies revealed that cells remained highly viable when treated with o.1\% PEG-TRAP for 2 days (Figure S2). Interestingly, an increase of activity was observed for cells treated with PEG-TRAP as proven by both cellular LDH and MTT assay. This is very likely due to the increase of cell proliferation as a consequence of molecular stimulation of PAR-1 receptors on $\mathrm{C}_{2} \mathrm{C}_{12}$ cells.


Figure 2. Platelet aggregation assay of whole blood mixed with PEG-TRAP and TRAP: $1 \mathrm{mM}$ [TRAP]; negative control: PEG-8MAL. A, real-time platelet aggregation curves of blood samples; B, the number of aggregation area. Data are presented in mean values and standard deviation (SD).

With this information in mind, we assessed the capability of PEG-TRAP conjugates for platelet activation via multiple electrode aggregometry (Multiplate ${ }^{\circledast}$ ). Multiplate ${ }^{\circledast}$ is a clinical-relevant tool to analyze the ability of patient-derived blood for platelet aggregation, which is a crucial step in the process of hemostasis. The principle is that the number of adherent platelets can be precisely measured by monitoring the electric signals on the electrode. As shown in Figure 2, both PEG-TRAP and TRAP solutions at equal peptide concentration $(1 \mathrm{mM})$ induced a rapid increase of electric signals that correspond to the extent of platelet aggregation. By contrast, the unconjugated polymer PEG-8MAL only brought negligible effects. Quantification of the key parameter (i.e., Aggregation Area in units [U]: the integrated area underneath the aggregation curve) evidenced that the number of units for PEG-TRAP, TRAP and PEG-8-MAL is 205, 238, and 8, respectively. These data show that the PEG-TRAP conjugates can induce platelet aggregation efficiently in a similar fashion as TRAP peptide alone, whereas the PEG-8-MAL precursors cannot.

To study the effects of PEG-TRAP on blood coagulation, rotational thromboelastometry (ROTEM) was utilized as a powerful tool to mechanically monitor the clotting kinetics. When citrated blood is re-calcified with $\mathrm{CaCl}_{2}$, coagulation is initiated and the kinetics of clot formation can be monitored in a quantitative fashion. A ROTEM test provides three important parameters: clotting time (CT) in s, the latency until the clot reaches a firmness of $2 \mathrm{~mm}$; clot formation time (CFT) in s, the time from CT until the clot reaches a firmness of $20 \mathrm{~mm}$; maximum clot firmness (MCF) in $\mathrm{mm}$, the absolute strength of the clot.

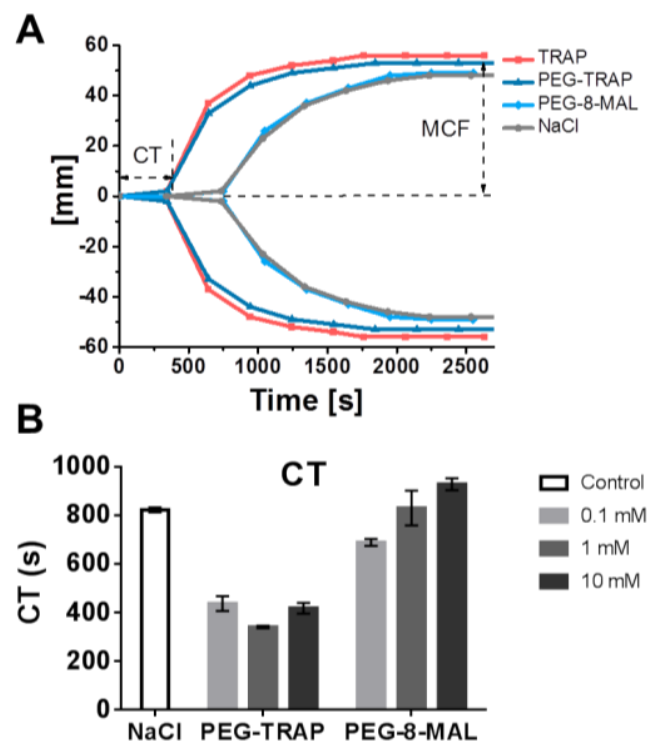

Figure 3. A, Clotting process of whole blood supplemented with different samples as monitored by ROTEM: 1 mM TRAP. B, clotting time (CT) of PEG-TRAP solutions of varying peptide concentration: $0.1 \mathrm{mM}, 1.0 \mathrm{mM}$, and $10 \mathrm{mM}$.

Figure $3 \mathrm{~A}$ shows the clotting curves of whole blood supplemented with either platelet-activating substances (TRAP, PEG-TRAP) or non-activating (PEG-8-MAL, NaCl) substances. The CT for a physiological coagulation $(\mathrm{NaCl})$ is ca. $800 \mathrm{~s}$. By contrast, the coagulation with $1 \mathrm{mM}$ PEGTRAP was significantly accelerated with a CT of ca. $400 \mathrm{~s}$, which is very comparable to that of TRAP at equal peptide concentration. The addition of PEG-8-MAL even induced a slight prolongation of the clotting process, presumably due to its side reactions with coagulation factors. Comparison in the absolute clot strength (Figure $\mathrm{S}_{3}$ ) show a similar trend: the platelet-activating PEG-TRAP conjugates 
increased the MCF from $45 \mathrm{~mm}$ to $\sim 50 \mathrm{~mm}$ as did the unconjugated TRAP control, whereas the PEG-8-MAL did not. Moreover, the effect of PEG-TRAP conjugates of varying concentration on coagulation was assessed (Figure 3B). It was found that the 1 mM PEG-TRAP was the most effective one by shortening the CT to 380 s (i.e., $\sim 45 \%$ of the $\mathrm{CT}$ of $\mathrm{NaCl}$ control). These data altogether demonstrated the high efficiency of PEG-TRAP for platelet activation and acceleration of blood coagulation.

Finally, we assessed whether the PEG-TRAP activated platelets present the characteristic activation marker $(\mathrm{CD} 62 \mathrm{P})$ via fluorescence-activated cell sorting (FACS). With this method, the extent of platelet activation is defined as the percentage of cells that co-express $C_{41}$ and $\mathrm{CD} 62 \mathrm{P}$ markers. As shown in Figure 4, there was only a very low extent of platelet activation (0.9\%), when whole blood was mixed with $\mathrm{NaCl}$. In comparison, there was a remarkable increase of platelet activation (59\%) after the addition of PEG-TRAP. The extent of platelet activation for TRAP was ca. $58 \%$. These experiments proved once more that the PEG-TRAP conjugates retain high efficiency for targeting PAR-1 receptors on platelets and for inducing platelet activation.
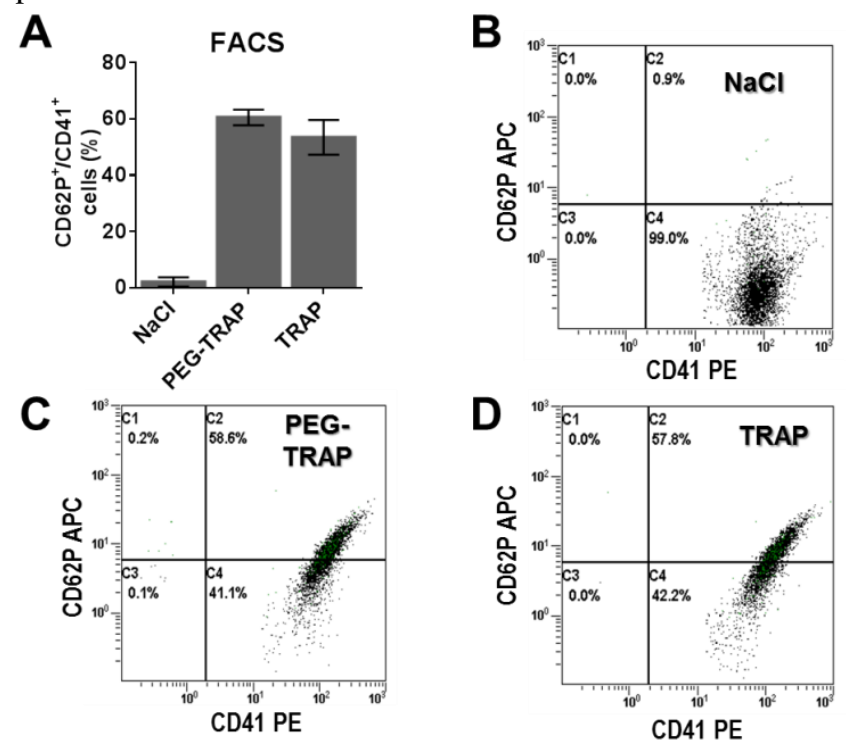

Figure 4. FACS analysis of platelet activation. A, percentage of $\mathrm{CD}_{2} \mathrm{P}^{+} / \mathrm{CD}_{41} 1^{+}$platelets after treatment with TRAP samples for $15 \mathrm{~min}$. B-D, representative dot-plots for the CD62 ${ }^{+} / \mathrm{CD}_{41^{+}}$ coexpression of blood samples treated with $\mathrm{NaCl}(\mathbf{B}), 1 \mathrm{mM}$ PEG-TRAP (C), and 1 mM TRAP (D).

In conclusion, a new class of synthetic polymer-peptide conjugates that can specifically activate platelets and promote hemostasis are designed and characterized. Unlike conventional plasma-derived fibrin/thrombin hemostats, PEG-TRAP is based on purely synthetic cell-compatible PEG matrices that avoid the risk of transporting bloodborne pathogens like viruses in potential surgical applications. The selected TRAP-functionalization approach is based on facile thiol-maleimide conjugation, which is also applicable to other synthetic or natural polymers bearing maleimide groups. We envision that PEG-TRAP conjugates can be used as soluble macromolecular agonists for activating platelets in hemostasis applications. Since PEGTRAP can activate platelets and promote platelet aggregation via the thrombin receptor PAR-1 without triggering fibrin formation, these molecularly engineered conjugates are promising candidates for the evaluation of platelet disorders and other platelet functions.

\section{ASSOCIATED CONTENT}

Supporting Information. Materials, experimental procedure, cytotoxicity analysis and additional ROTEM data are described in Supporting Information. This material is available free of charge via the Internet at http://pubs.acs.org."

\section{AUTHOR INFORMATION}

\section{Corresponding Authors}

Dr. Xiao-Hua Qin, Ludwig Boltzmann Institute for Experimental and Clinical Traumatology, Vienna, Austria ETH Zurich, Institute for Biomechanics, Zurich, Switzerland E-mail:xh.qin84@gmail.com; qinx@ethz.ch

Dr. Paul Slezak, Ludwig Boltzmann Institute for Experimental and Clinical Traumatology, Vienna, Austria E-mail: paul.slezak@trauma.lbg.ac.at

\section{Author Contributions}

The manuscript was written through contributions of all authors. All authors have given approval to the final version of the manuscript.

\section{Funding Sources}

This study was supported the Precision Polymer Materials Network of the European Science Foundation (ESF-P2M). X. Qin was supported by the SNSF Spark Award (no. 190345).

\section{ACKNOWLEDGMENT}

We would like to thank Anna Khadem for her invaluable support with blood experiments, Walter Dazinger (TU Wien) for the help with NMR measurements, Andreas Gössl and Christoph Schlimp for fruitful scientific discussion.

\section{ABBREVIATIONS}

TRAP, thrombin receptor-activating peptide; PEG, polyethylene glycol; ROTEM ${ }^{\circledR}$, rotational thromboelastometry; FACS, fluorescence-activated cell sorting.

\section{REFERENCES}

1. Lawson, J. H.; Murphy, M. P., Challenges for providing effective hemostasis in surgery and trauma. Seminars in Hematology 2004, 41, 55-64.

2. Behrens, A. M.; Sikorski, M. J.; Kofinas, P., Hemostatic strategies for traumatic and surgical bleeding. Journal of Biomedical Materials Research Part A 2014, 102 (11), 4182-4194.

3. Schneider-Barthold, C.; Baganz, S.; Wilhelmi, M.; Scheper, T.; Pepelanova, I., Hydrogels based on collagen and fibrin - frontiers and applications. BioNanoMaterials 2016, 17 (1-2).

4. Kumar, V. A.; Wickremasinghe, N. C.; Shi, S.; Hartgerink, J. D., Nanofibrous Snake Venom Hemostat. ACS Biomater Sci Eng 2015, 1 (12), 1300-1305.

5. Bertram, J. P.; Williams, C. A.; Robinson, R.; Segal, S. S.; Flynn, N. T.; Lavik, E. B., Intravenous hemostat: 
nanotechnology to halt bleeding. Science translational medicine 2009, 1 (11), 11 ra22.

$6 . \quad$ Chan, L. W.; Wang, X.; Wei, H.; Pozzo, L. D.; White, N. J.; Pun, S. H., A synthetic fibrin cross-linking polymer for modulating clot properties and inducing hemostasis. Science translational medicine 2015, 7 (277), 277 ra29.

7. Clemetson, K. J., Platelets and primary haemostasis. Thrombosis research 2012, 129 (3), 220-4.

8. $\quad$ Coughlin, S. R., How the protease thrombin talks to cells. Proceedings of the National Academy of Sciences 1999, 96 (20), 11023-11027.

9. Coughlin, S. R., Protease-activated receptors in hemostasis, thrombosis and vascular biology. Journal of thrombosis and haemostasis : JTH 2005, 3 (8), 1800-14.

10. Scarborough, R. M.; Naughton, M. A.; Teng, W.; Hung, D. T.; Rose, J.; Vu, T. K.; Wheaton, V. I.; Turck, C. W.; Coughlin, S. R., Tethered ligand agonist peptides. Structural requirements for thrombin receptor activation reveal mechanism of proteolytic unmasking of agonist function. J Biol Chem 1992, 267 (19), 131469.

11. Qin, X. H.; Labuda, K.; Chen, J.; Hruschka, V.; Khadem, A.; Liska, R.; Redl, H.; Slezak, P., Development of Synthetic Platelet-Activating Hydrogel Matrices to Induce Local Hemostasis. Advanced Functional Materials 2015, 25 (42), 6606-6617.

12. Fu, Y.; Kao, W. J., In situ forming poly(ethylene glycol)based hydrogels via thiol-maleimide Michael-type addition. Journal of Biomedical Materials Research Part A 2011, 98 A (2), 201-211.

13. Phelps, E. A.; Enemchukwu, N. O.; Fiore, V. F.; Sy, J. C.; Murthy, N.; Sulchek, T. A.; Barker, T. H.; Garcia, A. J., Maleimide cross-linked bioactive PEG hydrogel exhibits improved reaction kinetics and cross-linking for cell encapsulation and in situ delivery. Adv Mater 2012, 24 (1), 64-70, 2. 


\section{Table of Content (TOC)}

Hemostatic cell-compatible peptide conjugates based on multi-arm polyethylene glycol (PEG) are synthesized and characterized. These conjugates can specifically target the thrombin receptor on platelets, induce platelet activation, and accelerate blood coagulation by more than $\sim 50 \%$. These materials are promising for potential bio-surgical applications.

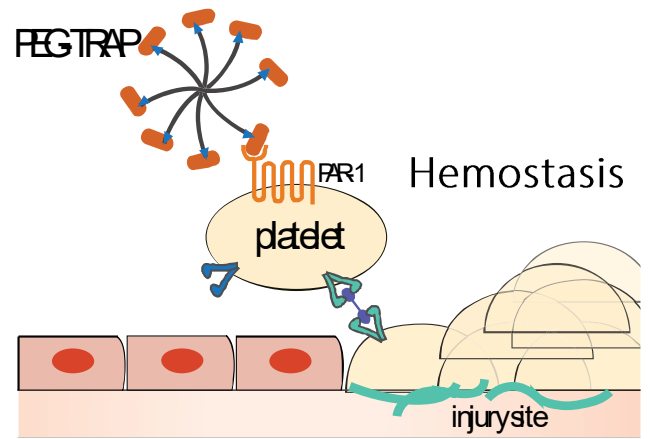

